\title{
Referente didáctico para la autoría de recursos educativos digitales en la Facultad de Estudios a Distancia de la Universidad Militar Nueva Granada (Colombia)*
}

Didactic reference for the authoring of digital educational resources in the Faculty of Distance Studies of the Universidad Militar Nueva Granada (Colombia)

Citar como: Esteban Ojeda, C. y Varela Vélez, O. I. (2020). Referente didáctico para la autoría de recursos educativos digitales en la Facultad de Estudios a Distancia de la Universidad Militar Nueva Granada (Colombia). CITAS, 6(1). https:/ /doi.org/10.15332/24224529.6361

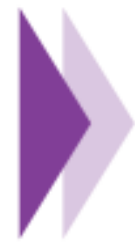

Catherine Esteban Esteban Ojeda ${ }^{1}$; Oscar Iván Varela Vélez ${ }^{2}$

1 Universidad Militar Nueva Granada 2 Universidad Militar Nueva Granada

Correo electrónico: yuri.esteban@unimilitar.edu.co 


\section{Resumen}

Este artículo describe la experiencia significativa de aprendizaje, lograda a través de los años, por el equipo administrativo y docente adscrito a la Dirección Académica de Desarrollo Multimedia (DADM) de la Facultad de Estudios a Distancia (Faedis) de la Universidad Militar Nueva Granada. Tiene la intención de condensar y guiar las principales premisas al momento de elaborar el material de estudio multimedia para la Faedis. Por lo tanto, se narran los principales hitos históricos y los lineamientos que conforman el manual de autor o guía didáctica útil para la creación de contenidos digitales educativos y curriculares en educación mediada por tecnología. Se tiene en cuenta cómo, para la construcción del componente didáctico del manual de autor, se contó con la experiencia pedagógica, comunicativa y tecnológica de las personas que han estado involucradas en los procesos, quienes no solo poseen el conocimiento especializado para el desarrollo multimedia, sino que cuentan con la experiencia significativa de interacción con estudiantes y docentes; esto enriquece notablemente el trabajo diario que realiza la Facultad de Estudios a Distancia. El presente artículo es referente para la línea de investigación de apropiación social y circulación del conocimiento, exactamente para la generación didáctica de contenido multimedia.

Palabras clave: recurso educativo digital, objeto virtual de aprendizaje, gestor de contenidos, diseño instruccional.

\section{Introducción}

El propósito principal de la creación del referente didáctico manual de autor- fue formar docentes, autores, pares disciplinares y otros actores educativos en cada uno de los momentos relevantes del proceso de elaboración del material de estudio o Recursos Educativos Digitales (RED). Este paso es el inicio fundamental para el proceso de construcción y elaboración de un recurso educativo digital, en el cual interviene un equipo multidisciplinar con diferentes perfiles tales como: autores o docentes expertos en el campo disciplinar, pedagogos, comunicadores, ingenieros de sistemas, diseñadores gráficos y multimedia, productores, auditores, docentes expertos en teorías y ámbitos del aprendizaje, así como profesionales calificados en el diseño de plataformas e\#learning, quienes deben contar con un contexto común que les permita, en su conjunto, lograr la integración de una producción intelectual que construye un autor docente.

De igual forma, con este artículo se pretende que la experiencia y recorrido realizado por los autores sirva a otros maestros y organizaciones dedicadas a la creación de recursos para el aprendizaje en línea con rigor y criterio académico.

\section{Justificación}

La Facultad de Estudios a Distancia, en su evolución procedimental, ha utilizado diversos manuales de autores como guía inicial para el desarrollo de los RED. En su inicio estos comprendían documentos escritos y manuales con lineamientos que, en muchas ocasiones, se tornaban confusos, dificultaban la narrativa educativa digital y la interacción con el equipo de desarrollo multidisciplinar que apoyaba la construcción de los recursos multimediales. Al reconocer esta dificultad se vio la necesidad de usar los diferentes saberes aprendidos en la creación de un recurso didáctico que permitiera a los autores, docentes y al

\section{Abstract}

This article describes the significant learning experience achieved over the years by the administrative and teaching team attached to the Academic Direction of Multimedia Development (DADM, in Spanish) of the Faculty of Distance Studies (Faedis, in Spanish) of the Universidad Militar Nueva Granada. It is intended to condense and guide the main premises when elaborating the multimedia study material for the Faedis. Therefore, it narrates the historical milestones and guidelines that make up the author's manual or didactic guide useful for the creation of educational and curricular digital content in technology\#mediated education. It is taken into account how, for the construction of the didactic component of the author's manual, it had the pedagogical, communicative and technological experience of the people who have been involved in the processes, who not only have the specialized knowledge for multimedia development, but also have significant experience of interacting with students and teachers; this significantly enriches the daily work carried out by the Faculty of Distance Studies. This article is a reference for the research line of social appropriation and circulation of knowledge, exactly for the didactic generation of multimedia content.

Keywords: digital educational resource, virtual learning object, content manager, instructional design.

equipo de desarrollo multimedia contar con lineamientos mínimos y ejemplificantes que ayudaran a la armonización en un mismo concepto, con la intencionalidad educativa para cada recurso de aprendizaje.

\section{Marcos de referencia y metodológico}

En el 2012, dentro de la Facultad de Estudios a Distancia, el proceso de creación de RED comprendía la contratación de autores expertos en su área disciplinar. En ese tiempo, los contenidos iniciales eran desarrollados por estos expertos en compañía de directores de programa, quienes se encargaban de la creación de un documento tipo libro que condensaba el saber hacia la construcción de un recurso educativo digital. Este documento contaba con todo el saber teórico y experiencias de los autores, pero requería de un gran trabajo de los diseñadores instruccionales en la interpretación y transformación del contenido inicial hacia la construcción de objetos virtuales de aprendizaje ${ }^{1}$; esto conllevó grandes esfuerzos y procesos que dificultaban la realización y entrega en tiempo de los RED. Debido a esta situación, se potenció el equipo de trabajo; se vincularon expertos en pedagogía y didáctica en ambientes virtuales de aprendizaje, iniciando con la optimización del proceso de creación de contenidos basados en referentes pedagógicos como: constructivismo, cognitivismo y la teoría de aprendizaje Gestalt: fundamentada en un entorno de eficiencia de tiempo y calidad, bajo la plataforma informativa de software educativo PLISE desarrollada por la Facultad de Estudios a Distancia (Jaramillo, 2010), basada en el software Adobe Authorware para su construcción, que posteriormente evolucionó a una solución sobre plataforma Adobe Flash, logrando así una evolución tecnológica y pedagógica para aquel momento.

Esta evolución y optimización de los procesos y tiempos llegó con un nuevo reto: la actualización y ampliación de la cobertura de la Facultad de Estudios a Distancia con nuevos programas, cuya meta era el desarrollo y actualización de 600 OVA. Esto 
conllevó a desarrollar una nueva estrategia de producción más eficiente, teniendo como base la metodología ADDIE (Branch, 2009) para el desarrollo de recursos didácticos, entendidos como la apropiación de diferentes metodologías y estrategias de enseñanza de aprendizaje por parte de los profesores y distintos actores partícipes de la creación de contenido multimedia, en el contexto de la formación docente. La didáctica es un referente que impulsa la reflexión de la acción de los profesores, idea que cobra mayor importancia dados los múltiples escenarios y modalidades de aplicación e intervención educativa (Díaz, 2009).

Entre 2013 y 2014, con el propósito de cumplir con esta importante misión, se amplió el equipo de trabajo y se estructuró un proceso metodológico con flujos de trabajo y controles de calidad eficientes, tanto en la elaboración conceptual, curricular y disciplinar como en la elaboración tecnopedagógica de los recursos, perfeccionando así la construcción del documento maestro en sinergia con el guion de medios interactivos para ser transferido al equipo de producción.

También en este año se optimizó el proceso de producción y visualización de los contenidos en una nueva plataforma desarrollada por el equipo de desarrollo multimedia denominada $E D D$, entorno de desarrollo digital, la cual permite la elaboración de la propuesta de diseño instruccional y la previsualización de contenidos para ser distribuido dentro del grupo de producción, reduciendo así tiempos y movimientos frente a fallas de comunicación o disonancias en el proceso. De este aplicativo se generaba una versión publicable del contenido para ser presentado a los estudiantes y docentes en la evolución de PLISE 3.0 (figura 1), pues para aquel momento se anunciaba la salida del mercado de los recursos educativos desarrollados en tecnología Flash y se veía la necesidad de acercar más el autor al producto final y al diseño instruccional.

Figura 1. Evolución de la plataforma para la gestión de contenidos multimedia

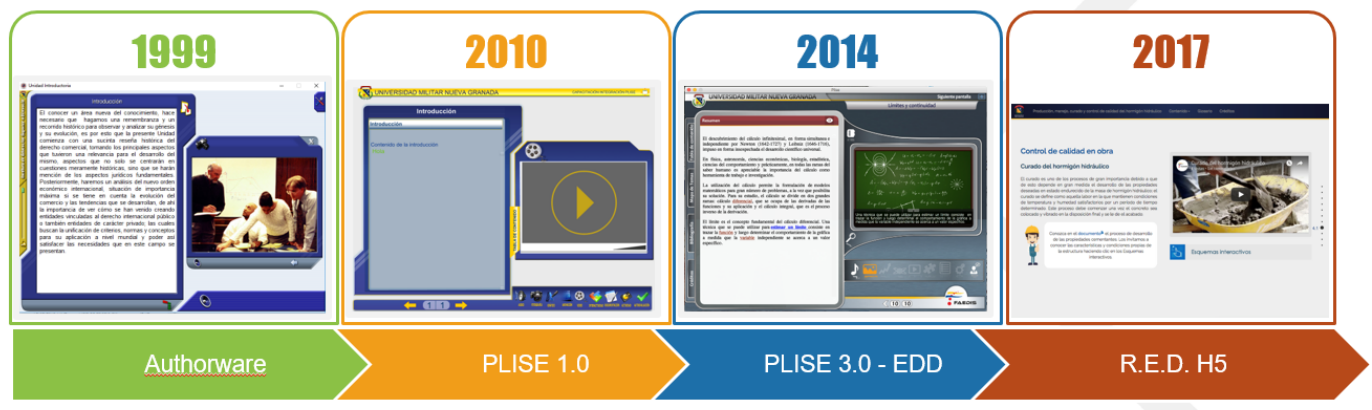

Fuente: elaboración propia.

La primera versión en digital del manual de autor inició su producción en el 2015, el cual contaba con el acompañamiento de los autores dentro de un ambiente virtual (aula virtual) creado para la dinamización de la producción intelectual de los RED. Esta aula contó inicialmente con una carpeta guía para trabajo de autoría con orientaciones en documentos de Word y PDF, de formatos editables de documento maestro, contenido programático de asignatura, formato de autoevaluación, formato de interactividades y guion básico de video. En su momento, el manual de autor ilustraba de manera académica y pedagógica cómo iniciar la producción intelectual teniendo en cuenta el área de la asignatura, el plan de estudios, sus créditos y su carácter teórico, teórico-práctico o práctico (Universidad Militar Nueva Granada, 2015). También se invitaba a los autores a realizar una revisión del decálogo de competencias transversales que se trabajaba en facultad y las competencias disciplinares específicas correspondientes a la asignatura que se deseaba desarrollar; esto con el fin de crear una obra didáctica (RED) robusta y válida para la apropiación de conocimiento y el desarrollo de habilidades. En esta dinámica de construcción se identificaron varias dificultades, entre otras: docentes sin descarga académica en su plan de trabajo para la creación de contenidos; ausencia de acompañamiento de un líder disciplinar por parte de los programas que validara la producción intelectual a manera de par disciplinar; el diseño instruccional realizaba la corrección de estilo e interpretación de las ideas del autor de acuerdo con sus preconcepciones, debido a que no se contaba por parte del autor con los ejemplos didácticos y plausibles de cómo podrían quedar sus producciones intelectuales descritas en el cuadro de medios utilizado en aquel momento (figura 2). 
Figura 2. Cuadro de medios en documento maestro

\begin{tabular}{|c|c|c|c|c|c|c|}
\hline Tipo de medio. Seleccione el medio & $\begin{array}{l}\text { Video } \\
\text { ( ) }\end{array}$ & Imagen & $\begin{array}{l}\text { Infografia } \\
\text { () }\end{array}$ & $\begin{array}{l}\text { Animación } \\
\text { ( ) }\end{array}$ & $\begin{array}{l}\text { Texto } \\
\text { (] }\end{array}$ & $\begin{array}{l}\text { Otro } 0 \\
\text { Cuál }\end{array}$ \\
\hline \multicolumn{7}{|l|}{ Nombre: } \\
\hline \multicolumn{7}{|c|}{ Relato referente al medio (El medio trata de $\ldots$ y presenta... ) } \\
\hline \multicolumn{7}{|c|}{$\begin{array}{l}\text { Descripción detallada del medio para producirlo o elaborarlo en la UMNG: Se requiere un medio que contenga ... y permita al } \\
\text { estudiante interactuar de la siguiente manera..., ver guion en la carpeta }\end{array}$} \\
\hline
\end{tabular}

Fuente: tomada de Varela y Esteban (2014).

A partir de estas dificultades presentadas se tomó la decisión de construir un herramienta didáctica que fuera referente para el desarrollo de los Recursos Educativos Digitales de la Facultad de Estudios a Distancia, la cual debería contemplar: ejemplos de medios ya realizados, situar al autor en el producto esperado, lineamientos pedagógicos, didácticos y comunicativos, estructura de actividades de aprendizaje y autoevaluaciones, diseño instruccional básico, estudios de caso, pedagogía activa, modelos de citación de fuentes bibliográficas, lecturas complementarias, entre otros. Todo esto acogiendo los lineamientos pedagógicos institucionales y de la Facultad de Estudios a Distancia, los cuales cobran valor desde un aprendizaje centrado en el estudiante. En este los enfoques base son el constructivismo y el cognitivismo, y lo trasversal se sustenta en dos teorías de aprendizaje: la Gestalt y el conectivismo, tal como lo propone Díaz (2009): "es perentorio que se asuman las propuestas a la luz de la didáctica: de ningún modo puede trasladarse al espacio del aula la tecnología sin un apropiado conocimiento y de los principios didácticos” (p. 131).

De este modo, en 2016 y 2017 se inició la producción intelectual del manual de autor ya en versión $\mathrm{HTML}^{2}$ (Esteban, 2017), con el propósito principal de formar docentes, autores, pares disciplinares y directores de programa, en cada uno de los momentos relevantes del proceso de elaboración del material de estudio (tipo RED), que son puestos al servicio de los estudiantes en la modalidad de educación a distancia. Este manual cuenta, en primera instancia, con algunos de los conceptos y apropiaciones iniciales que los autores o expertos disciplinares deben tener presentes a la hora de crear, desarrollar, validar y evaluar la producción intelectual en los diferentes programas académicos de la Universidad Militar Nueva Granada. Los tres principales referentes conceptuales son: recursos educativos digitales, material de estudio y objetos virtuales de aprendizaje (OVA).

El manual de autor muestra las etapas para la construcción del material de estudio (Esteban, 2017) (figura 3), la apropiación del contenido programático, la elaboración del documento maestro y la construcción de medios en documento maestro (Esteban, Obando y Camacho, 2017) ${ }^{3}$, tales como: texto, locución, fotografía, video, infografía, animaciones, interactividades, lecturas complementarias, hipervínculos, preguntas orientadoras y cápsulas informativas. 
Figura 3. Etapas para la construcción de material de estudio

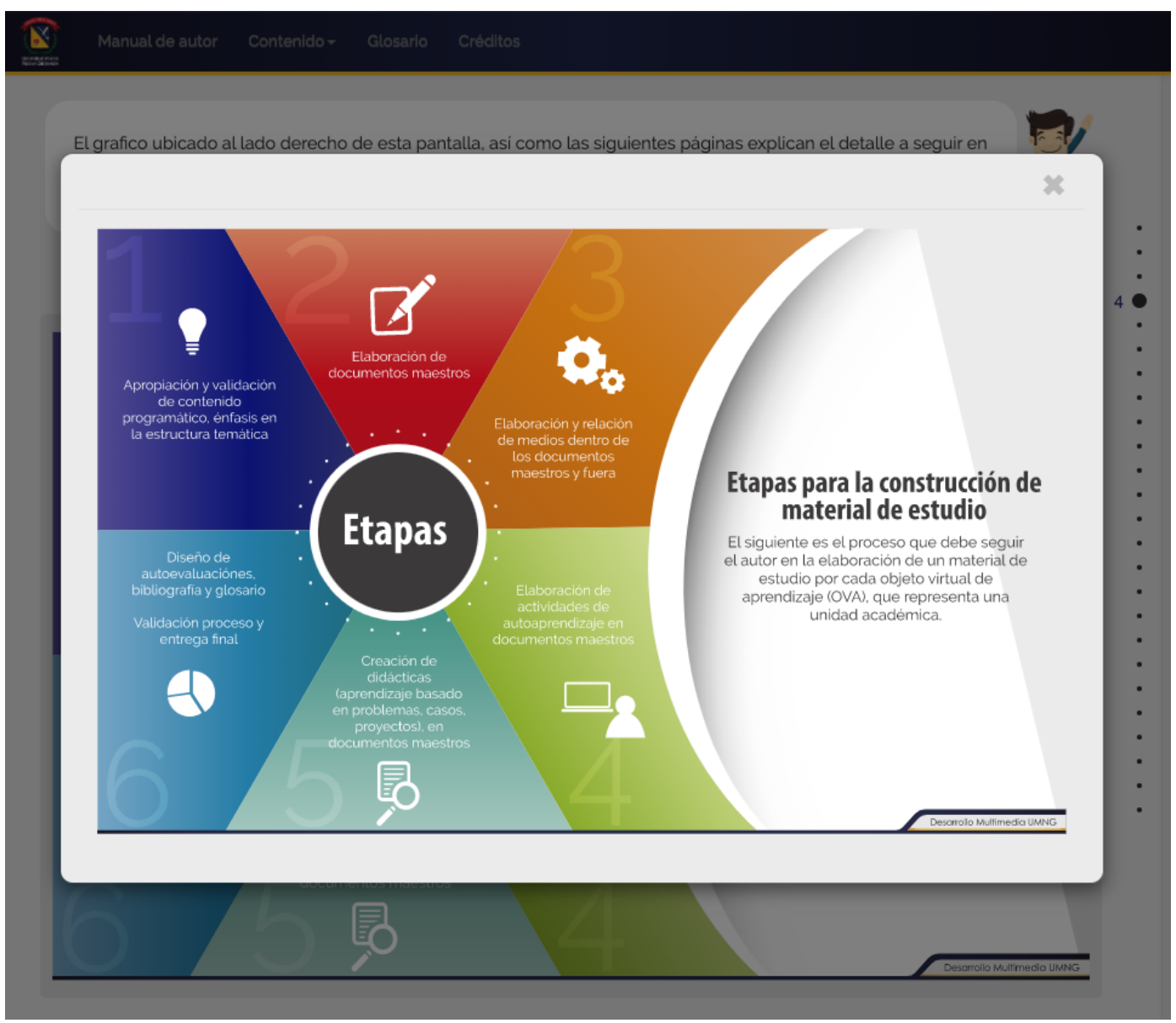

Fuente: Universidad Militar Nueva Granada (2017).

Otro de los componentes importantes del documento maestro y que marca el principal diferenciador fue crear escenarios para la construcción de actividades de autoaprendizaje como mediación didáctica para estimular la actividad del estudiante y evitar lo que podría ser un largo y tedioso monólogo del docente, promoviendo así la interacción activa con el estudiante de manera directa. Los ejercicios construidos hasta el momento son variados en contenido, duración, forma, competencia o desempeño a desarrollar, y lugar o medio de presentación; esto garantiza una ayuda pedagógica autoinstructiva.

El docente autor hace uso de este manual con una guía para la construcción de pedagogías activas tales como el diseño de un caso, un problema, una situación, una realidad simulada en contexto o retos, entre muchas otras. Estas, a su vez, se trabajan con el equipo de la Dirección Académica de Desarrollo Multimedia $(2017)^{4}$ para validar si su salida virtual es una animación, un video, un videoquiz, una interactividad, una simulación, un diseño en 2D, una gamificación, etc. Finalmente, el referente didáctico declara un espacio importante a la autoevaluación y realimentación como escenario de actividad compleja y amplia, realizada durante todo el proceso formativo profesional y que busca medir y valorar el nivel de apropiación de los conocimientos y el desarrollo de procesos, habilidades y competencias de los estudiantes.

Es así como este RED construido en HTML se ha convertido en el referente didáctico y pedagógico que guía al núcleo profesoral de la Facultad de Estudios a Distancia de la Universidad Militar Nueva Granada en la construcción de sus contenidos educativos digitales. De este modo, el manual de autor es una orientación didáctica para la estructuración de todo proceso en donde se analicen, organicen y guíen las estrategias de enseñanza de aprendizaje mediadas por la tecnología. "De allí la insistencia de volver la mirada a la didáctica para interpretar la enseñanza, su complejidad y diversidad" (Díaz, 2009).

En 2018, 2019 y 2020 se ha trabajado sobre esta base procedimental del manual de autor para impulsar la construcción de nuevos paradigmas de la educación, como son los Cursos Masivos Abiertos en Línea (MOOC), cursos de corta duración tipo empresarial y webinar, con el objetivo, para el 2021, de contener los principios para la creación de cursos basados en inteligencia artificial hacia un proceso y proyecto de educación hiperpersonalizada. 
Es por ello que la Dirección Académica de Desarrollo Multimedia ha desarrollado una nueva plataforma de trabajo colaborativo, que permitirá la construcción y designación de proyectos de RED hacia y por diferentes frentes de trabajo colaborativo. Lo anterior con una visión de reúso y aprovechamiento de los datos e información que allí se generen orgánica e inorgánicamente, sobre tiempos, movimientos, evolución, así como patrones de comportamiento de los usuarios, frecuencia de uso y comportamientos, con el fin de evolucionar en conjunto la experiencia de usuario, y así anticiparse a los nuevos requerimientos y detectar falencias o errores en el momento justo, al contar siempre con información relevante y oportuna, que ajusta el manual de autor en beneficio de nuestra comunidad académica.

Esta experiencia permite afirmar que se han mejorado los tiempos para la construcción de materiales de estudio, al pasar de casi un año en autoría y cuatro meses más en la construcción de un guion de medios por parte de autores para ser entregado al equipo de construcción del OVA en el 2012, a casi cuatro meses en la elaboración del nuevo documento de autor, el cual incluye la autoría, el guion de medios, estrategias de aprendizaje, autoevaluaciones, actividades de aprendizaje y cápsulas informativas, lo cual se transfiere al equipo de construcción del OVA. Esto sin contar la disminución de reprocesos entre diseñadores gráficos e integradores y diseñadores instruccionales, por la facilidad de interpretación y la visualización previa de los contenidos preformateados en el aplicativo EDD, lo que aumenta la calidad y la cantidad de los contenidos digitales producidos en la actualidad.

\section{Resultados obtenidos}

La tabla 1 muestra la evolución de tiempos de producción y autoría por cada cuatro OVA en promedio y su tiempo en meses, lo cual evidencia la reducción del tiempo en la construcción de los OVA para las disciplinas de los programas en la Faedis.

Tabla 1. Evolución de tiempos de producción y autoría por cada cuatro OVA en promedio

\begin{tabular}{|l|l|l|l|l|l|}
\hline Año & Autoría & Guion & $\begin{array}{c}\text { Diseño } \\
\text { instruccional y } \\
\text { adaptación del } \\
\text { guion }\end{array}$ & $\begin{array}{c}\text { Producción e } \\
\text { integración }\end{array}$ & $\begin{array}{l}\text { Tiempo } \\
\text { (meses) }\end{array}$ \\
\hline $2012-2013$ & 8 & 3 & 1 & 1.5 & $13 \cdot 5$ \\
\hline $2014-2015$ & 4 & 2 & 1 & 1 & 8 \\
\hline $2016-2019$ & 4 & 1 & 1 & 6 \\
\hline
\end{tabular}

Fuente: tomada de la Sección de Desarrollo Multimedia, de Varela (2020),

Dirección Académica de Desarrollo Multimedia. Universidad Militar Nueva Granada.

La tabla 2 da cuenta de los recursos construidos por programa de acuerdo con el año, y muestra la diferencia frente a programas nuevos, tal es el caso del programa de Ingeniería informática. 
Tabla 2. Recursos construidos entre 2012 y 2019

\begin{tabular}{|c|c|c|c|c|c|c|c|c|c|}
\hline \multirow{2}{*}{ Programa } & \multicolumn{9}{|c|}{ Información de OVA publicados por año } \\
\hline & 2012 & 2013 & 2014 & 2015 & 2016 & 2017 & 2018 & 2019 & Total \\
\hline $\begin{array}{l}\text { Administración de } \\
\text { empresas }\end{array}$ & $\mathrm{o}$ & $\mathrm{o}$ & 9 & 10 & 104 & 32 & 53 & 19 & $\mathbf{2 2 7}$ \\
\hline $\begin{array}{l}\text { Contaduría } \\
\text { pública }\end{array}$ & $\mathrm{o}$ & 4 & 10 & & 15 & 16 & 10 & 13 & 68 \\
\hline $\begin{array}{l}\text { Administración de } \\
\text { la seguridad y } \\
\text { salud ocupacional }\end{array}$ & 54 & 27 & 65 & 19 & 27 & 3 & 6 & 5 & 206 \\
\hline $\begin{array}{l}\text { Relaciones } \\
\text { internacionales y } \\
\text { estudios políticos }\end{array}$ & 29 & 7 & 4 & $\mathrm{O}$ & $\mathrm{o}$ & 15 & 44 & $\mathrm{o}$ & 99 \\
\hline Ingeniería civil & & 16 & 8 & o & $\mathrm{o}$ & 5 & 35 & $\mathrm{O}$ & 64 \\
\hline $\begin{array}{l}\text { Ingeniería } \\
\text { industrial }\end{array}$ & 27 & 36 & 52 & 38 & 5 & 17 & 11 & 19 & 205 \\
\hline $\begin{array}{l}\text { Ingeniería } \\
\text { informática }\end{array}$ & $\mathrm{o}$ & $\mathrm{o}$ & $\mathrm{o}$ & 21 & & 20 & 27 & 55 & 123 \\
\hline Posgrados & 18 & 13 & o & 7 & 39 & 8 & 5 & 9 & 99 \\
\hline Otros OVA & o & o & 15 & 11 & 6 & 1 & 3 & 2 & 38 \\
\hline Total & 128 & 103 & 163 & 106 & 196 & 117 & 194 & 122 & 1129 \\
\hline
\end{tabular}

Fuente: tomada de la Dirección Académica de Desarrollo Multimedia, de Varela (2019), Universidad Militar Nueva Granada.

\section{Experiencias en la construcción con los autores}

Los autores identifican en este nuevo escenario de producción intelectual un rigor y exigencia que parte desde la construcción del Syllabus a nivel de comité de currículo, el acompañamiento del par disciplinar experto en el saber, el par pedagógico y el equipo de la Dirección Académica de Desarrollo Multimedia (DADM), que orienta, guía y dinamiza todo el proceso.

En la construcción, los autores se enfrentan a posibles desconocimientos de la modalidad virtual: ¿cómo trasladar su conocimiento y experiencia a nuevas didácticas con apropiación de tecnología para el aprendizaje? ¿Cómo pasar del texto a otros medios digitales? Para ello, el equipo de la DADM ha creado un espacio de ambiente virtual de aprendizaje en donde ellos son estudiantes y sus pares pedagógicos y disciplinares cumplen un rol de profesor, mentor y tutor de todo el proceso.

Al final, los autores están a gusto y satisfechos con sus desarrollos y al ser entregado su material de estudio indican que han potenciado su docencia y ya no se desgastan tanto en la presentación de clase, sino que acompañan con mayor tiempo el proceso de enseñanza de aprendizaje.

\section{Experiencias en la elaboración de los recursos}

A los autores les cuesta en un principio pensar en la construcción de didácticas activas que lleven a la interacción y realimentación con el material de estudio y que su saber pueda ser un material autoinstructivo, o quizás temen que al escribir su saber pierdan su legitimidad docente. Es por esto que el equipo de pedagogía trabaja con ellos en sesiones síncronas o asíncronas, en donde orientan la construcción de cada uno de los recursos, actividades y evaluaciones que componen la obra didáctica tipo OVA. Los docentes suben sus construcciones a través de guiones o formatos prediseñados al aula virtual creada y desde esta instancia se generan líneas de asesoría gráfica, comunicativa, pedagógica o multimedia. Los profesores manifiestan, con la construcción de cada medio digital, audiovisual, multimedia, entre otros, una gran oportunidad de difundir su saber con la comunidad académica. De igual manera, señalan que sus tutorías actuales son más didácticas, ilustrativas, amenas y los estudiantes reconocen la calidad del material. De hecho, algunos de ellos han compartido esto en sus empresas para que puedan estudiar con nosotros o se mejoren los procesos organizacionales con base en estas obras académicas.

\section{Conclusiones}

La educación a distancia y mediada por tecnología requiere de un trabajo arduo en la producción intelectual de materiales de estudio significativos, situados y adaptados al contexto, y tienen el reto de facilitar rutas de aprendizaje que declaren un mejor acceso al conocimiento y un eficiente aprovechamiento del tiempo del estudiante, quien debe asumir la responsabilidad de su propio aprendizaje. 
Este manual de autor ilustra los principales lineamientos al momento de elaborar el material multimedia para la Facultad de Estudios a Distancia (Faedis) de la Universidad Militar Nueva Granada. Las normas y consejos están fundamentadas en la experiencia pedagógica y tecnológica de las personas que trabajan para la Dirección Académica de Desarrollo Multimedia, quienes no solo poseen el conocimiento especializado, sino que además cuentan con la experiencia de trabajo directo con el núcleo de profesores. Esto enriquece notablemente el quehacer diario de la Facultad de Estudios a Distancia en la Universidad Militar Nueva Granada.

El desafío y reto que asume el docente de la Universidad Militar u otra institución de educación al seguir el manual de autor es plasmar y permitir la mediación de sus conocimientos a través de la construcción de un objeto virtual de aprendizaje, que concibe con claridad los conceptos y la pertinencia pedagógica, mediante el uso de un lenguaje caracterizado por la claridad, la sencillez y la contundencia, a fin de lograr efectivos desempeños de aprendizaje en cada uno de los estudiantes o aprendices a su cargo. De igual forma, es un referente para la línea de investigación de apropiación social y circulación del conocimiento, puntualmente para la generación didáctica de contenido multimedia.

Como resultado del manual de autor, el proceso de elaboración de recursos educativos digitales de calidad se ha optimizado, ha reducido los tiempos y evitado momentos de confusión en interpretaciones diversas por los diferentes miembros que conforman el equipo de producción de OVA a casi la mitad del tiempo empleado inicialmente. Esto aumenta la producción y acerca los momentos de autoría, construcción y publicación, así se mantiene un ritmo y actualización de los contenidos ofrecidos a los estudiantes.

\section{Referencias}

Branch, R. M. (2009). Instructional Design: The ADDIE Approach. Springer.

Esteban, C. (2017). Manual de autor 2017. Universidad Militar Nueva Granada.

Facultad de Estudios a Distancia, Universidad Militar Nueva Granada. (2017). Cuadro de medios en documento maestro [video]. YouTube. https://youtu.be/RpVbx1D38Ws

Díaz, A. (2009). Pensar la didáctica. Amorrortu.

Dirección Académica de Desarrollo Multimedia. (2017). Creación de didácticas activas en documento maestro (estudio de caso). Universidad Militar Nueva Granada. http://virtual.umng.edu.co/distancia/ecosistema/ovas/ dadm/manual_autor/unidad_1/medios/interactividades/pat19 12/pat19_12.html

Jaramillo, J. (2010). Plataforma informativa de software educativo PLISE. Facultad de Estudios. Universidad Militar Nueva Granada.

Universidad Militar Nueva Granada. (2015). Reglamento General Estudiantil de pregrado. Acuerdo 02 de 2015 (p. 14). Universidad Militar Nueva Granada.

Universidad Militar Nueva Granada. (2017). Cuadro de medios en documento maestro [interactividad multimedia]. http://virtual.umng.edu.co/distancia/ecosistema/o vas/dadm/manual_autor/unidad_1/medios/interactividades/pat 13/pat13.html
Notas

$*$

Texto presentado en el IV Foro Internacional de Investigación en Ambientes Virtuales de Aprendizaje

1 Uno o varios recursos educativos digitales conforman un objeto virtual de aprendizaje en la Universidad Militar Nueva Granada.

2 De este modo, en 2016 y 2017 se inició la producción intelectual del manual de autor, que está disponible, de manera libre, en el OVA, en Manual de autor, publicado por la Universidad Militar Nueva Granada en http://virtual.umng.edu.co/distancia/ecosistema/odi n/odin_desktop.php?path=Li4vb3\%20Zhcy9kYWRtL 21hbnVhbF9hdXRvci91bmlkYWRfMS8=\#slide_1\% 207.

3 Véase el video Cuadro de medios en documento maestro (Facultad de Estudios a Distancia, Universidad Militar Nueva Granada, 2017).

4 Para una ejemplificación didáctica del proceso de trabajo del autor docente en la construcción de un OVA ver: Facultad de Estudios a Distancia (2017) Manual de Autor. Universidad Militar Nueva Granada. http://virtual.umng.edu.co/distancia/ecosiste $\mathrm{ma} /$ ovas/dadm/manual_autor/unidad_1/medios/intera ctividades/pat19_12/pat19_12.html.

\section{(c) (i) (ㅇ)}

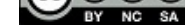

